\title{
Comparação da sobrevivência e dos fatores prognósticos em pacientes com adenocarcinoma gástrico T2 e T3
}

\section{Comparison of survival and prognostic factors in patients with gastric adenocarcinoma in $T 2$ and $T 3$}

\author{
Patrícia Campos Juca - ACBC-RJ'; Laercio Lourenço - TCBC-SP2; Rubens Kesley - TCBC-RJ'; \\ Eduardo Linhares Riello de Mello - TCBC-RJ'; Ivanir Martins de Oliveira ${ }^{3}$; José Humberto Simốes Correa - TCBC-RJ'
}

R E S U M O

\begin{abstract}
Objetivo: Comparar a sobrevivência e os fatores prognósticos, após o tratamento cirúrgico de pacientes com adenocarcinoma gástrico que compromete a camada muscular própria (T2), e de pacientes cujo tumor invade a subserosa (T3). Métodos: Estudo retrospectivo de 122 pacientes com câncer gástrico invadindo a muscular própria e subserosa, submetidos ao tratamento cirúrgico no período de janeiro de 1997 a dezembro de 2008 e acompanhados até dezembro de 2010. Foram analisadas variáveis demográficas, cirúrgicas e anatomopatológicas. Resultados: Dos 122 pacientes, 22 (18\%) foram excluídos da análise final porque apresentaram: margem positiva, mortalidade pós-operatória, segundo tumor primário e menos de 15 linfonodos na peça cirúrgica. Entre os 100 pacientes incluídos, 75 apresentavam tumores com invasão da muscular própria (T2) e 25 com invasão da subserosa (T3). A sobrevivência global foi $83,8 \%$, sendo $90,6 \%$ no T2 e 52,1\% no T3. Na análise univariada apresentaram significância: metástase linfonodal $(p=0,02)$, tamanho do tumor $(p=0,000)$, estadiamento patológico do tumor $(p=0,000)$, estadiamento patológico linfonodal $(p=0,000)$ e estadiamento por grupos da classificação TNM-UICC/AJCC, $2010(p=0,000)$ Na análise multivariada, os fatores prognósticos independentes foram o tamanho do tumor e o estadiamento patológico linfonodal (pN). Conclusão: O comprometimento linfonodal e o tamanho do tumor são fatores prognósticos independentes nos tumores com invasão da muscular própria e nos tumores com invasão da subserosa. O T2 apresenta menor tamanho, menor taxa de linfonodos metastáticos e consequentemente, melhor prognóstico que o T3.
\end{abstract}

Descritores: Prognóstico. Neoplasias. Neoplasias gástricas. Adenocarcinoma. Taxa de sobrevida.

\section{INTRODUÇÃO}

A incidência do câncer gástrico (CG) vem diminuindo mundialmente desde 1950. Entretanto, sua agressividade, malignidade e, consequentemente, seu prognóstico permanecem inalterados, representando a segunda maior causa de morte por câncer, com 628.000 (12,1\%) óbitos/ano1. No Brasil, segundo o Instituto Nacional de Câncer² (INCA), a estimativa de incidência do CG para o ano de 2011 apontava para 21.500 casos novos da doença, sendo 13.820 em homens (64,3\%) e 7680 em mulheres (35,7\%), correspondendo a um risco estimado de 14 novos casos para cada 100.000 homens e de oito para cada 100.000 mulheres, consolidando-se como quinto tumor maligno em incidência e segundo do trato gastrointestinal.

O adenocarcinoma gástrico ( $A G$ ) é o tipo histológico mais frequente (95\%), que se origina no epitélio da mucosa gástrica e progressivamente envolve toda a parede do estômago até atingir a serosa e estruturas adja- centes. O tratamento cirúrgico permanece como a única modalidade de tratamento curativo ${ }^{3,4}$ e a extensão da ressecção depende da avaliação pré e intraoperatória da localização do tumor, do grau de penetração do tumor na parede do estômago, da invasão de órgãos adjacentes e de metástase linfonodal ${ }^{5,6}$.

A identificação de fatores prognósticos do $A G$ é importante para estabelecer o estadiamento e determinar as estratégias terapêuticas. O tumor que invade as camadas mucosa e submucosa ( $\mathrm{T} 1$ ), independente do comprometimento linfonodal, é classificado como câncer gástrico precoce (CGP), com sobrevivência em cinco anos de 93,5\% dos pacientes operados, sendo de $72,8 \%$ naqueles com linfonodos positivos, e de $95,6 \%$, quando não há metástases linfonodais. Quando o tumor ultrapassa a camada submucosa e invade a muscular própria (T2), classifica-se como câncer gástrico avançado (CGA), porém é considerado um estádio intermediário de progressão tumoral entre o CGP e o CGA, com melhor prognóstico e sobrevivên-

Trabalho realizado no Programa de Pós-Graduação em Ciência Cirúrgica Interdisciplinar, da Universidade Federal de São Paulo - Escola Paulista de Medicina - SP-BR.

1. Cirurgião do Instituto Nacional de Câncer, Rio de Janeiro-RJ-BR; 2. Professor Adjunto do Departamento de Cirurgia da Universidade Federal de São Paulo-SP-BR; 3. Chefe do Serviço de Patologia do Instituto Nacional de Cancer - Rio de Janeiro-RJ-BR. 
cia em cinco anos ${ }^{7-14}$. Essa categoria (T2), pouco frequente, representa $8 \%$ a $18 \%$ dos CG ressecados no Japão ${ }^{13}$.

A classificação TNM do American Joint Committee on Cancer (AJCC) de 2010, estabelece critérios de estadiamento baseados na invasão do tumor na parede gástrica, comprometimento linfonodal e metástases à distância. Os tumores são agrupados em categorias de acordo com a invasão na parede do estômago (T), números de linfonodos comprometidos $(\mathrm{N})$ e presença de metástases à distância (M). Em 1998, o College of American Pathologists passou a recomendar a subclassificação do T2 em T2a - invasão da muscular própria, e T2b - invasão da subserosa, para permitir melhor avaliação prognóstica e de sobrevivência nesses pacientes $^{15}$. No início deste estudo, utilizou-se a sexta edição da classificação TNM-UICC/AJCC ${ }^{16}$, que definia essa subdivisão, recomendada pelo College of American Pathologists para o T2, entretanto, sem que houvesse modificação no estadiamento dos grupos que incluem as lesões T2 (estágios IB, II e IIIA), ou seja, pT2aN0 e pT2bN0 estágio I e pT2aN1 e pT2bN1 estágio II. Apenas o comprometimento linfonodal determinava a mudança de estádio ${ }^{16}$.

Em 2010, foi publicada a sétima edição da classificação TNM-UICC/AJCC ${ }^{17}$, separando em categorias e estádios diferentes, os tumores restritos à muscular própria e aqueles que invadem a subserosa, T2 e T3, respectivamente. O T2a permanece na categoria T2 e o T2b passa para a categoria T3. O estadiamento patológico linfonodal (pN) também foi modificado, sendo N1 de um a dois linfonodos comprometidos, N2 de três a seis, N3a de sete a 15 e N3b de 16 ou mais linfonodos comprometidos. No estadiamento por grupos, as categorias T2 e T3 foram separadas, T2N0 é estádio IB, T3N0 e T2N1 estádio IIA, T3N1 e T2N2 estádio IIB, T3N2 e T2N3 estádio IIIA e T3N3 estádio IIIB. Esta atual classificação exclui os tumores da junção esofagogástrica (JEG) ou que se originam no estômago a menos de $5 \mathrm{~cm}$ da JEG e a invadem. Esses tumores, de pior prognóstico, são agora estadiados como o adenocarcinoma esofágico ${ }^{17}$. Essa separação do T2 e T3 em grupos de estadiamento diferentes corrobora que o CG T2 apresenta prognóstico diferente do T3. A identificação dos fatores prognósticos relacionados ao T2 é importante porque, nesse estádio intermediário, o câncer pode ser curável com o procedimento cirúrgico adequado que inclui a linfadenectomia D2.

Poucos são os estudos publicados avaliando os fatores prognósticos em pacientes com $T 2^{8,10,13,14}$. Vários estudos sugerem um prognóstico favorável para o T2 com sobrevivência em cinco anos, próxima ao T18,18-21. Outras séries referem que a subclassificação do T2 (T2/T3) não tem valor na presença de linfonodos metastáticos, já que o que determina o prognóstico dos pacientes é o comprometimento linfonodal ${ }^{10,22}$.

Este estudo tem por objetivo comparar, após o tratamento cirúrgico, a sobrevivência e os fatores prognósticos de pacientes com adenocarcinoma gástrico T2, e T3.

\section{MÉTODOS}

Foram comparados 122 pacientes portadores de AG, com invasão da muscular própria (T2) e invasão da subserosa (T3). Todos os pacientes foram tratados no Serviço de Cirurgia Abdominopélvica do Hospital do Câncer I, INCA, entre janeiro de 1997 e dezembro de 2008, e acompanhados até dezembro de 2010. O estudo foi aprovado pelos Comitês de Ética em Pesquisa do Instituto Nacional de Câncer, registro número 69/2009, e da Universidade Federal de São Paulo sob o registro 1484/2009.

Dos 122 doentes, 22 foram excluídos da análise final, pois um apresentava margem comprometida pela neoplasia no estudo histopatológico definitivo $(0,8 \%)$, quatro evoluíram para o óbito nos 30 primeiros dias de pósoperatório $(3,2 \%)$, quatro apresentaram um segundo tumor primário (3,2\%), e 13 tiveram menos de 15 linfonodos ressecados $(10,6 \%)$

Dos 100 pacientes incluídos, 75 apresentavam tumores com invasão da muscular própria (T2), e 25, com invasão da subserosa (T3), de acordo com a sétima edição da classificação TNM-UICC/AJCC, 2010 17. O tratamento cirúrgico radical seguiu os critérios da Japanese Gastric Cancer Association (JGCA) ${ }^{5}$ de acordo com a localização do tumor. A gastrectomia subtotal (GST) foi realizada nos tumores do terço distal e a gastrectomia total (GT), nos tumores do terços proximal. Os tumores localizados no terço médio foram submetidos à GST ou GT na dependência da distância da borda proximal do tumor até a JEG.

A linfadenectomia foi realizada com base na localização do tumor no estômago, incluindo os linfonodos do nível I e/ou II, de acordo com o relato do cirurgião e conforme os critérios da classificação japonesa ${ }^{5}$, sendo considerada adequada a amostragem linfonodal mínima de 15 linfonodos ressecados. A ressecção foi considerada curativa (RO) na ausência de resíduo tumoral macroscópico ou microscópico, com margens, proximal e distal, livres de neoplasia no exame anatomopatológico durante o ato operatório, e na ausência de doença à distância .

As variáveis demográficas estudadas foram o sexo e a idade. Os pacientes foram divididos em duas faixas etárias, com 60 anos ou menos e com mais de 60 anos, determinadas pela mediana e média da idade.

As variáveis cirúrgicas incluíram o tipo de gastrectomia, total ou subtotal, e a extensão da linfadenectomia realizada, nível 1 (D1) ou nível 1 e 2 (D2), descritas pelo cirurgião.

As variáveis anatomopatológicas macroscópicas analisadas de acordo com as médias e medianas encontradas foram o tamanho do tumor ( $d^{\prime \prime} 5 \mathrm{~cm}$ ou $>5 \mathrm{~cm}$ ) e o número de linfonodos ressecados (d"33 linfonodos e >33 linfonodos). $O$ aspecto macroscópico do tumor foi analisado conforme a classificação de Borrmann ${ }^{23}$ para CGA, incluindo a categoria zero de acordo com a classificação japonesa para CGP (JGCA $)^{5}$, quando a aparência macroscópica do tumor sugeria ao patologista tumor pre- 
coce. A localização no estômago (terços proximal, médio ou distal) foi baseada na classificação japonesa da JGCA ${ }^{5}$.

As variáveis anatomopatológicas microscópicas incluíram a invasão do tumor na parede gástrica, camada muscular própria versus subserosa; o grau de diferenciação celular, segundo a classificação de Broders ${ }^{24}$, moderadamente e bem diferenciado vs. pouco diferenciado; a presença ou ausência de invasão venosa, linfática e neural; a presença ou ausência de linfonodo comprometido; o número de linfonodos comprometidos pela neoplasia e o grupamento por estágios, conforme a sétima edição da classificação TNM-UICC/AJCC ${ }^{17}$.

\section{Análise Estatística}

Os dados obtidos no estudo dos 100 pacientes foram analisados, utilizando-se: 1) análise descritiva da amostra, pela distribuição de frequências simples e percentuais, medianas, médias aritméticas, desvios padrões (DP) e intervalos de confiança (IC); 2) teste exato de Fisher, de modo bicaudal; 3) método de Kaplan-Meier para análise de sobrevivência, aplicando-se o teste de Log Rank para comparação das curvas de sobrevivência. Óbitos não oncológicos não foram censurados; 4) método de Cox Proportional Hazards para análise multivariada dos fatores prognósticos, Harzard ratio pelo teste Z; e 5) o nível de significância de $5 \%$ de probabilidade. As variáveis sem significância foram assinaladas com a sigla NS.

\section{RESULTADOS}

A estimativa global de sobrevivência em cinco anos foi $81,8 \%$ dos casos, com média de seguimento de $124 \pm 6,3$ meses (95\% IC, 111 a 136 meses) pode ser vista na figura 1.

A média e mediana da idade foram 60 anos (25 a 78 anos no T2 e 42 a 83 anos no T3). Quanto ao sexo, houve equivalência: $53 \%$ eram do sexo masculino e $47 \%$ do sexo feminino. No sexo masculino predominou a quinta década de vida. A idade $(p=0,78)$ e o sexo $(p=0,48)$ não interferiram significativamente no prognóstico dos pacientes.

A GST foi realizada em $74 \%$ (59\% no T2 e 15\% no T3) e a GT em 26\% (16\% no T2 e 10\% no T3). Dezessete pacientes foram submetidos à linfadenectomia D1, e, em todos, foram encontrados mais de 15 linfonodos na peça cirúrgica. Quanto ao prognóstico, as variáveis tipo de operação e extensão da linfadenectomia não interferiram significativamente na sobrevivência em cinco anos.

As variáveis anatomopatológicas macroscópicas estudadas foram a localização, o aspecto macroscópico e o tamanho do tumor, e o número de linfonodos ressecados. Verificou-se diferença significativa no prognóstico dos pacientes classificados como T2 e T3 apenas em relação ao tamanho do tumor $d^{\prime \prime} 5 \mathrm{~cm}$ e $>5 \mathrm{~cm}(\mathrm{p}=0,00)$.

O tumor localizava-se no terço distal do estômago em 57\% dos pacientes; no terço médio, em $31 \%$; e no terço proximal, em 12\%. A média de linfonodos ressecados foi 33, com variação de 15 a 75 linfonodos. A média e mediana do tamanho do tumor foi de $5 \mathrm{~cm}$. Pacientes com tumores $\mathrm{d}^{\prime \prime} 5 \mathrm{~cm}$ apresentaram sobrevivência em cinco anos de $94,6 \%$, e com $>5 \mathrm{~cm}$, de $69 \%(p=0,000)$ (Figura 2). Quando analisado o tamanho de acordo com o estadiamento patológico do tumor ( $\mathrm{pT}$ ), também houve diferença significativa. No pT2 d" $5 \mathrm{~cm}(59 \%)$ e $>5 \mathrm{~cm}(41 \%)$, a sobrevivência foi $96,5 \%$ e $82,1 \%$, respectivamente $(p<0,005)$. No pT3 d" $5 \mathrm{~cm}(32 \%)$ e $>5 \mathrm{~cm}(68 \%)$, a sobrevivência foi $80 \%$ e $45 \%$, respectivamente $(p<0,005)$.

$\mathrm{Na}$ análise do grau de penetração do tumor na parede gástrica (pT), a sobrevivência em cinco anos foi significativamente maior no pT2 $(90,6 \%)$, que no T3 $(52,1 \%),(p=0,000)$ (Figura 3).

Metástases linfonodais ocorreram em 52\% dos pacientes com sobrevivência, em cinco anos, de $72,4 \%$, que, quando comparada à sobrevivência de $90,3 \%$ dos pacientes com linfonodos negativos, apresentou significância estatística $(p<0,02)$. O estadiamento patológico linfonodal - pN - também demonstrou diferença significativa na so-

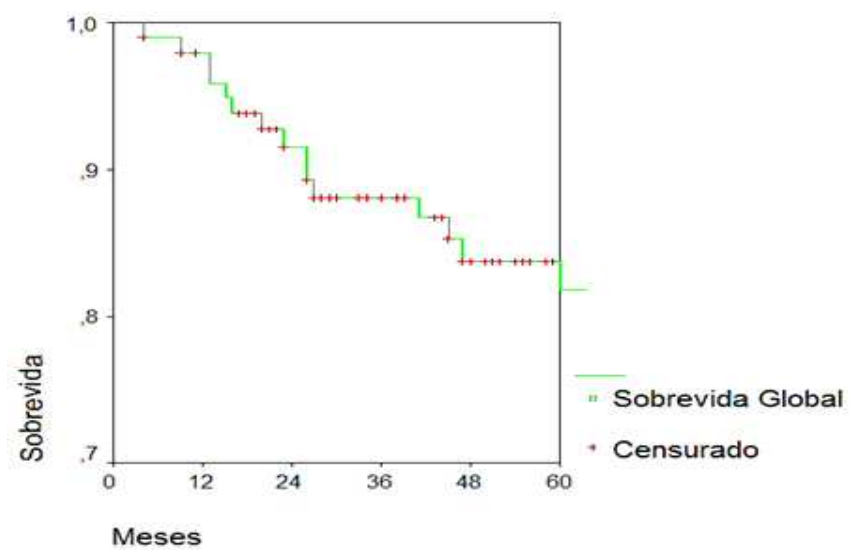

Figura 1 - Curva de sobrevivência em cinco anos de 100 pacientes com AG T2 e T3. Método de Kaplan-Meier.

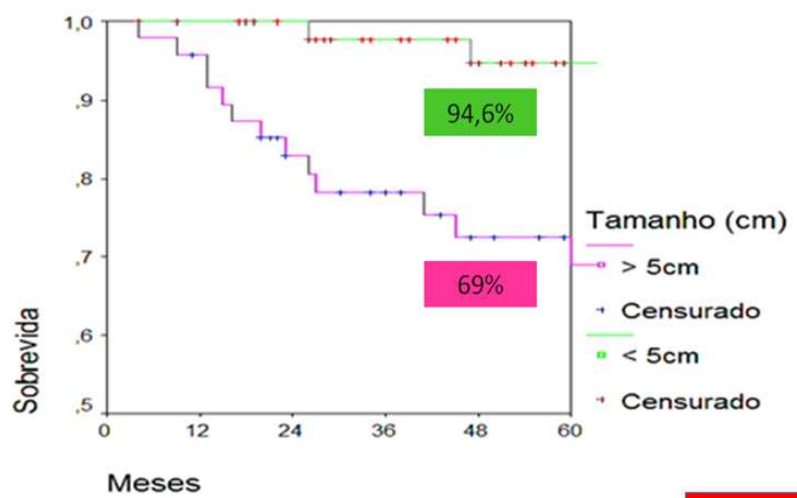

Figura 2 - Curva de sobrevivência em cinco anos conforme o tamanho, demonstrando que houve diferença significativa de prognóstico nos tumores $d^{\prime \prime} 5 \mathrm{~cm}$ e $>5 \mathrm{~cm}$ $(p=0,000)$. Método de Kaplan-Meier. 
brevivência dos pacientes $(p=0,000)$ (Figura 4). Quando analisado o pN de acordo com o pT2 e com o pT3, também houve diferença significância na sobrevivência $(p<0,001)$ (Tabela 1), o que também ocorreu no estadiamento por grupos TNM-UICC/AJCC ${ }^{17},(p=0,000)$.

O grau de diferenciação celular do tumor e presença ou ausência de infiltração venosa, linfática e neural não foram significativos.

Dos 100 pacientes estudados, 16\% apresentaram recidiva da doença durante o seguimento, dos quais dois ainda estão vivos, com evidência de doença, um com metástase óssea e o outro com metástase em linfonodos do pedículo hepático. A taxa de recidiva foi significativamente maior no T3. Dos 75 pacientes com T2, oito $(10,7 \%)$ apresentaram recidiva, sendo seis à distância (fígado e ossos), um em linfonodos e um no peritônio. Oito (32\%) pacientes com T3 apresentaram recidivas, sendo cinco à distância (fígado, ossos e pulmão), um em linfonodos e dois peritoneais $(p<0,0171)$. Ocorreram 17 (17\%) óbitos, 14 em decorrência do CG e três por causas não relacionadas ao CG. Todos os pacientes foram seguidos por um período médio de 124 meses, mínimo de quatro meses e máximo de 152 meses.

Na análise univariada, os fatores prognósticos associados à sobrevivência foram o tamanho do tumor $(p=0,000)$, a invasão do tumor na parede gástrica - $p T$ $(p=0,000)$, a presença de metástase linfonodal $(p=0,02)$, o número de linfonodos comprometidos $-\mathrm{pN}-(\mathrm{p}=0,000)$ e o estadiamento por grupo da classificação TNM-UICC/AJCC ${ }^{17}$ $(p=0,000)$. A análise multivariada demonstrou que os fatores prognósticos independentes foram tamanho $(p<0,001)$ e comprometimento linfonodal $(p<0,016)$ (Tabela 2$)$.

\section{DISCUSSÃO}

Existem muitos estudos sobre o CGA, porém, poucos avaliam os fatores prognósticos dos pacientes com T2, a maioria deles com casuística semelhante a do presente estudo e seus resultados são controversos. A maior casuística foi o estudo coreano de Park et al. ${ }^{19}$, com 442 pacientes. Também não está claro quais desses fatores prognósticos são relevantes no $\mathrm{T}^{10}{ }^{10}$. O possível melhor prog- nóstico do T2 tem determinado criticas a sua definição de CGA. O T2 é considerado como prognóstico intermediário entre o CGP e CGA ${ }^{7-14}$

A identificação de fatores prognósticos relacionados ao CG é fundamental para a avaliação prognóstica e decisão da melhor estratégia terapêutica, cirúrgica e adjuvante. Estudos demonstram que a invasão do tumor na parede do estômago e o Encontramos estudos que tam-

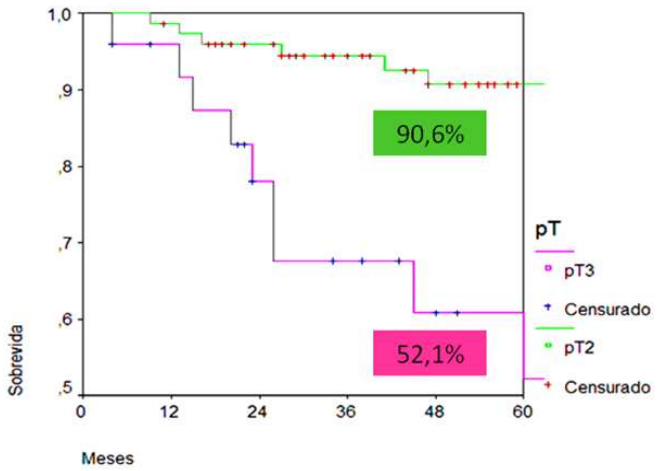

Figura 3 - Curva de sobrevivência em cinco anos conforme estadiamento patológico do tumor, demonstrando que houve diferença significativa no prognóstico dos pacientes de acordo com o pT $(p=0,000)$. Método de Kaplan-Meier.

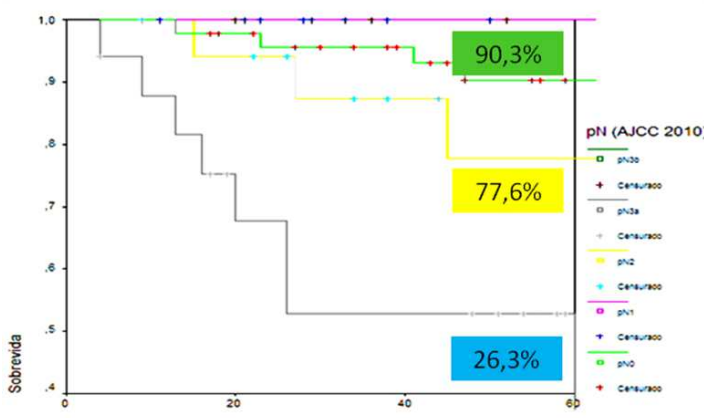

SOBREVIDA

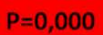

Figura 4 - Curva de sobrevivência em cinco anos conforme o estadiamento patológico linfonodal, demonstrando que houve diferença significativa no prognóstico dos pacientes de acordo com o pN. $(p=0,000)$. Método de Kaplan-Meier.

Tabela 1 - Análise do estadiamento patológico linfonodal no T2 e T3.

\begin{tabular}{lrcrrrrrr}
\hline pN & \multicolumn{1}{c}{$\mathrm{n}$} & $\mathrm{pT2}(\%)$ & ES $\%$ & $\mathrm{Cl} 95 \%$ & $\mathrm{n}$ & $\mathrm{pT3}(\%)$ & $\mathrm{ES} \%$ & $\mathrm{IC} 95 \%$ \\
\hline pN0 & 44 & 58,7 & 92 & $126-151$ & 4 & 16 & 66,7 & $29-76$ \\
pN1 & 9 & 12 & vivos & - & 5 & 20 & vivo & - \\
pN2 & 11 & 14,7 & 89 & $79-111$ & 7 & 28 & 62,5 & $42-85$ \\
pN3a & 8 & 10,7 & 75 & $36-68$ & 9 & 38 & 38,1 & $18-50$ \\
pN3b & 3 & 4,0 & vivos & - & 0 & - & - & - \\
Total & 75 & 100 & - & - & 25 & 100 & - & - \\
\hline
\end{tabular}

Legenda: $n$ (número de casos); IC (intervalo de confiança); ES (estimativa de sobrevivência). 
Tabela 2 - Análise univariada de sobrevivência conforme as características clinicopatológicas dos 100 pacientes com adenocarcinoma gástrico T2 e T3.

\begin{tabular}{|c|c|c|c|c|c|c|c|c|c|}
\hline \multirow[t]{2}{*}{ Variáveis } & \multirow[t]{2}{*}{ Casos } & \multirow[t]{2}{*}{ Óbitos } & \multicolumn{2}{|c|}{ Vivos } & \multirow[t]{2}{*}{ Meses } & \multirow[t]{2}{*}{ DP } & \multirow[t]{2}{*}{ IC $(95 \%)$} & \multicolumn{2}{|c|}{ ES } \\
\hline & & & $\mathrm{n}$ & $\%$ & & & & $\%$ & $p<$ \\
\hline $\begin{array}{l}\text { Total } \\
\text { Idade: }\end{array}$ & 100 & 17 & 83 & 83 & 124 & 6,3 & $111-136$ & 81,8 & - \\
\hline$<60$ anos & 51 & 8 & 43 & 84,3 & 125 & 8,7 & $108-142$ & 82,1 & 0,780 \\
\hline $\begin{array}{l}\text { > } 60 \text { anos } \\
\text { Sexo: }\end{array}$ & 49 & 9 & 40 & 81,7 & 113 & 7,5 & $93-128$ & 81,0 & \\
\hline Feminino & 53 & 10 & 43 & 81,1 & 112 & 7,8 & 96- 127 & 80,0 & 0,480 \\
\hline $\begin{array}{l}\text { Masculino } \\
\text { Cirurgia: }\end{array}$ & 47 & 7 & 40 & 85,1 & 126 & 9,2 & $108-144$ & 84,0 & \\
\hline GST & 74 & 13 & 61 & 82,4 & 113 & 6,3 & $101-126$ & 81,2 & 0,920 \\
\hline $\begin{array}{l}\text { GT } \\
\text { Borrmann: }\end{array}$ & 26 & 4 & 22 & 84,6 & 130 & 10,1 & $110-150$ & 83,8 & \\
\hline 0 & 29 & 3 & 26 & 89,6 & 126 & 7,0 & $112-140$ & 88,4 & 0,290 \\
\hline I & 5 & 2 & 3 & 60 & 67 & 20,8 & $26-108$ & - & \\
\hline II & 22 & 5 & 17 & 77,2 & 95 & 9,03 & 77- 112 & 80,0 & \\
\hline III & 37 & 6 & 31 & 83,7 & 117 & 13,3 & $91-143$ & 81,2 & \\
\hline IV & 7 & 1 & 6 & 85,7 & 74 & 9,1 & $56-92$ & - & \\
\hline Tamanho: & & & & & & & & & \\
\hline$<5 \mathrm{~cm}$ & 52 & 2 & 50 & 96,1 & 146 & 4,1 & $138-154$ & 94,6 & 0,000 \\
\hline $\begin{array}{l}>5 \mathrm{~cm} \\
\text { Localização: }\end{array}$ & 48 & 15 & 33 & 68,7 & 96 & 8,8 & 79- 114 & 69,0 & \\
\hline Proximal & 12 & 1 & 11 & 91,6 & 140 & 11,8 & 116- 163 & 91,6 & 0,720 \\
\hline Médio & 31 & 5 & 26 & 83,8 & 112 & 11,2 & $90-134$ & 84,2 & \\
\hline Distal & 57 & 11 & 46 & 80,7 & 111 & 6,7 & $98-124$ & 78,8 & \\
\hline Espessura: & & & & & & & & & \\
\hline$<0,9 \mathrm{~cm}$ & 55 & 6 & 49 & 89 & 133 & 7,8 & $117-148$ & 89,2 & 0,070 \\
\hline $\begin{array}{l}>0,9 \mathrm{~cm} \\
\text {.linfonodos. }\end{array}$ & 45 & 11 & 34 & 75,5 & 103 & 8,4 & 86- 119 & 72,4 & \\
\hline$<33$ & 59 & 10 & 49 & 83 & 125 & 7,6 & $110-140$ & 80,3 & 0,920 \\
\hline$>33$ & 41 & 7 & 34 & 82,9 & 92 & 5,2 & 82- 103 & 83,6 & \\
\hline Grau diferença: & & & & & & & & & \\
\hline $\mathrm{G} 1 / \mathrm{G} 2$ & 34 & 7 & 27 & 79,4 & 97 & 6,9 & $83-111$ & 79,9 & 0,700 \\
\hline $\begin{array}{l}\text { G3 } \\
\text { Invasão pT: }\end{array}$ & 66 & 10 & 56 & 84,8 & 125 & 8,2 & 109-141 & 82,9 & \\
\hline $\mathrm{T} 2$ & 75 & 8 & 67 & 89,3 & 134 & 6,1 & $121-146$ & 90,6 & 0,000 \\
\hline $\begin{array}{l}\text { T3 } \\
\text { Inf. Venosa: }\end{array}$ & 25 & 9 & 16 & 64 & 63 & 7,7 & $48-79$ & 52,1 & \\
\hline Sim & 19 & 4 & 15 & 78,9 & 75 & 8,0 & $60-91$ & 73,3 & 0,360 \\
\hline $\begin{array}{l}\text { Não } \\
\text { Inf. Linfática: }\end{array}$ & 81 & 13 & 68 & 83,9 & 126 & 6,6 & $113-139$ & 83,4 & \\
\hline Sim & 63 & 13 & 50 & 79,3 & 106 & 7,3 & $91-120$ & 78,1 & 0,180 \\
\hline $\begin{array}{l}\text { Não } \\
\text { Inf. Perineural: }\end{array}$ & 37 & 4 & 33 & 89,1 & 136 & 7,4 & $122-151$ & 87,4 & \\
\hline Sim & 45 & 10 & 35 & 77,7 & 117 & 9,5 & $98-136$ & 78,9 & 0,330 \\
\hline Não & 55 & 7 & 48 & 87,2 & 121 & 6,1 & $109-133$ & 84,0 & \\
\hline $\mathrm{pN}:$ & & & & & & & & & \\
\hline 0 & 48 & 5 & 43 & 89,5 & 136 & 6,6 & $123-149$ & 90,3 & 0,000 \\
\hline 1 & 14 & 0 & 14 & 100 & - & - & $-\quad-$ & & \\
\hline 2 & 18 & 4 & 14 & 77,7 & 87 & 8,3 & $71-104$ & 77,6 & \\
\hline $3^{a}$ & 17 & 8 & 9 & 52,9 & 41 & 6,1 & $29-53$ & 26,3 & \\
\hline $3 b$ & 5 & 0 & 5 & 100 & - & - & - & - & \\
\hline Estadio: & & & & & & & & & \\
\hline $\mathrm{lb}$ & 44 & 4 & 40 & 90,9 & 139 & 6,3 & $129-151$ & 92,0 & 0,000 \\
\hline lia & 13 & 1 & 12 & 92,3 & 109 & 8,2 & $93-125$ & 90,9 & \\
\hline lib & 16 & 2 & 14 & 87,5 & 97 & 6,1 & $85-109$ & 91,6 & \\
\hline IIla & 18 & 4 & 14 & 77,7 & 67 & 6,7 & $54-80$ & 73,4 & \\
\hline $\mathrm{Illb}$ & 9 & 6 & 3 & 33,3 & 34 & 8,1 & $18-50$ & 0 & \\
\hline
\end{tabular}

Nota: Teste de Log-Rank; Graus de Liberdade (GL).Legenda: M (média); DP (desvio padrão); IC (intervalo de confiança); ES (estimativa de sobrevivência em cinco anos). 
bém utilizaram o maior diâmetro do tumor para avaliar seu tamanho, com medianas de 3,5, 3,6 $6^{19}$ e 4,2 $\mathrm{cm}^{21}$. Vários estudos ${ }^{19,20,22,25}$ encontraram diferença significativa relacionada ao tamanho do tumor na análise univariada. Fotia et al. ${ }^{10}$ analisando tumores $\mathrm{d}^{\prime \prime} 2 \mathrm{~cm}$, de 2 a $4 \mathrm{~cm}$, e $>4 \mathrm{~cm}$ não encontraram diferença significativa na sobrevivência em cinco anos. No presente estudo o tamanho do tumor foi fator prognóstico independente, $58,7 \%$ do T2 eram d" $5 \mathrm{~cm}$ e $68 \%$ do T3 $>5 \mathrm{~cm}$.

O AG com invasão da camada muscular própria apresenta melhor prognóstico que o tumor que invade a subserosa $a^{7,9,11,12,14,19,20,22,25,26}$, sendo fator prognóstico independente em vários estudos ${ }^{14,19,20,26}$. No presente estudo a sobrevivência do T2 foi significativamente melhor do que a do T3, 90,6\% e 52,\%, respectivamente. Quando analisado $\mathrm{PT}$ com outras variáveis prognósticas, como a presença de metástase linfonodal, a invasão do tumor na parede gástrica não foi fator prognóstico independente. Sarela et al. ${ }^{22}$ também encontraram diferença significativa na sobrevivência entre T2 e T3 (64\% vs. 46\%, $p=0,005)$, entretanto, quando o estadiamento linfonodal era adequado, com mais de 15 linfonodos ressecados, os autores encontraram resultados diferentes: a sobrevivência nos pacientes N0 foi semelhante no T2 e T3 $(90 \%$ vs. $86 \%, p=0,8$ ) e nos do N1, a sobrevivência não foi significativamente diferente no T2 e T3 (56\% vs. 44\%, $p=0,3$ ). Fotia et al. ${ }^{10}$, em estudo incluíndo pacientes com menos de 15 linfonodos ressecados, também não encontraram diferença na sobrevivência do T2 e T3 (74\% vs. 67\%, $\mathrm{p}=0,2)$. Park et al. ${ }^{19}$, entretanto, encontraram diferença entre o T2 e T3, independente do comprometimento linfonodal, em estudo incluindo apenas pacientes com mais de 15 linfonodos ressecados (85\% vs. 56\%, $p<0,001)$. No estudo de Nitti et al. ${ }^{26}$, o pT foi fator prognóstico independente, o T2 apresentou prognóstico significativamente melhor do que o T3, com sobrevivência em cinco anos de $73 \%$ e $31 \%$, respectivamente.

No presente estudo, metástases linfonodais ocorreram em $52 \%$ dos pacientes, com diferença significativa na sobrevivência, 90,3\% e 72,4\%, respectivamente, com linfonodos negativos e positivos $(p<0,02)$. As metástases linfonodais foram mais frequentes no T3 (84\%). Apenas 16\% dos pacientes T3 apresentaram linfonodos negativos. Em T2, 59\% apresentaram linfonodos negativos e $41 \%$ positivos. O comprometimento linfonodal também foi fator prognóstico na análise univariada de vários estudos 8,10,12,19,25. No estudo de Sarela et al. ${ }^{22}$, dois terços do T3 estavam associados à metástase linfonodal comparado com apenas metade do T2 $(p<0,001)$.

A estratificação dos pacientes de acordo com o comprometimento patológico linfonodal $(\mathrm{pN})$, também demonstrou diferença significativa $(p=0,000)$. O T3 apresentou maior número de linfonodos metastáticos, que foi progressivamente maior no N1 $(20 \%)$, N2 (28\%) e N3 (36\%). Sobrevivência no T2 foi melhor do que no T3, de acordo com o pN. No T2N0 a sobrevivência foi $92,6 \%$ e no
T3N0, 66,67\%. Todos os pacientes T2N1 e T3N1 estavam vivos até o término do estudo. No T2N2 e T3N2 a sobrevivência foi $89 \%$ vs. $62,5 \%$ e no T2N3a e T3N3a foi $45 \%$ vs. $38 \%$, respectivamente. Os pacientes T2N3b estavam vivos até o término do estudo e não havia pacientes com T3N3b. O pN foi fator prognóstico independente. No estudo de Sarela et al. ${ }^{22}$, as categorias pN2 e pN3 corresponderam a um quarto do T3 e a apenas 3\% do T2. A sobrevivência no pN0, pN1, pN2 e pN3 foi 83\%, 44\%, $11 \%$ e $0 \%$, respectivamente. O pN foi o único fator prognóstico independente nesse mesmo estudo.

A análise do estadiamento TNM-UICC/AJCC ${ }^{17}$, 2010, também demonstrou diferença significativa na sobrevivência em cinco anos, de acordo com os grupos de estádios. Os pacientes estádios IB, $\| \mathrm{A}$ e $\| \mathrm{B}$, que correspondem ao T2NO-N2 e T3NO-N1, apresentaram sobrevivência semelhante de $92 \%, 90,9 \%$ e 91,6\%, respectivamente, e nos estádios IIIA e IIIB (T2N3 e T3N1-N3) a sobrevivência foi $73,4 \%$ e $0 \%$, respectivamente. Dois estudos avaliaram a classificação TNM-UICC/AJCC, 2010, no CG. Ahn et al..$^{28}$ encontraram melhor categorização por grupos na sétima edição, principalmente relacionada ao T2 e T3, N1 e N2. Kim et al. ${ }^{29}$ também encontraram meIhor separação por subgrupos de estadiamento, com diferentes prognósticos.

Os tumores com invasão da muscular própria apresentam melhor prognóstico que os tumores que invadem a subserosa, e isso ocorre porque quanto mais profunda a invasão do tumor na parede do órgão, maior a taxa de linfonodos metastáticos. Sasako et al. ${ }^{30}$ demonstraram que o comprometimento linfonodal estava presente em $47 \%$ dos tumores que invadem a muscular própria e em $64 \%$ dos tumores que invadem a subserosa.

O estômago possui um sistema linfático bem desenvolvido e a disseminação linfática é a mais comum. Os tumores gástricos restritos à camada muscular própria apresentam menor taxa de metástase linfonodal, diminuindo o risco de disseminação, por isso apresentam melhor prognóstico que os tumores que invadem a subserosa.

Apesar dos avanços da Oncologia, o CG ainda é uma doença com prognóstico reservado, entretanto, nos últimos anos a detecção da doença em estádios mais precoces, a sistematização do tratamento cirúrgico radical e o surgimento da terapia adjuvante, tem aumentado a sobrevivência dos pacientes. É possível que, com aumento da utilização da endoscopia digestiva alta, a melhoria do sistema público de saúde e o desenvolvimento das condições socioeconômicas do país, o número de pacientes com CG restrito à muscular própria (T2) seja maior, melhorando efetivamente o prognóstico e sobrevivência.

Neste estudo, o comprometimento linfonodal e os tumores maiores que $5 \mathrm{~cm}$ em seu maior diâmetro foram os fatores que determinaram o prognóstico de pacientes com câncer gástrico que invade a muscular própria e a subserosa. O AG T2 apresentou menor tamanho e menor número de linfonodos metastáticos que o T3, o que deter- 
minou o melhor prognóstico e sobrevivência em cinco anos dos pacientes com AG com invasão da camada muscular própria do estômago quando comparados com aqueles com comprometimento da subserosa.

\title{
A B S T R A C T
}

\begin{abstract}
Objective: To compare the survival and prognosis after surgical treatment of patients with gastric adenocarcinoma which extends to the muscular layer (T2), and patients whose tumor invades the subserosa (T3). Methods: This was a retrospective study of 122 patients with gastric cancer invading the muscularis propria and subserosa, undergoing surgical treatment from January 1997 to December 2008 and followed-up until December 2010. We analyzed demographic, surgical and pathological variables. Results: Of the 122 patients, 22 (18\%) were excluded from the final analysis because they showed: positive margin or less than 15 lymph nodes in the surgical specimen, early postoperative mortality and second primary tumors. Among the 100 patients included, 75 had tumors inveding the muscularis propria (T2) and 25 with extension to the subserosa (T3). Overall survival was 83.8\%, and $90.6 \%$ for T2 and $52.1 \%$ or T3. Univariate analysis showed statistical significance in: lymph node metastasis $(p=0.02)$, tumor size $(p=0.000)$, tumor pathological stage $(p=0.000)$, lymph node pathologic stage $(p=0.000)$ and staging by classification of groups TNM-UICC/AJCC, 2010 $(p=0.000)$. In multivariate analysis, independent prognostic factors were tumor size and lymph node pathological staging ( $\mathrm{pN}$ ). Conclusion: The lymph node status and tumor size are independent prognostic factors in tumors with invasion of the muscularis propria and in tumors with invasion of subserosa. T2 lesions have smaller size, lower rate of lymph node metastasis and therefore better prognosis than T3.
\end{abstract}

Key words: Prognosis. Neoplasms. Stomach neoplasms. Adenocarcinoma. Survival rate.

\section{REFERÊNCIAS}

1. Parkin DM, Bray F, Ferlay J, Pisani P. Global cancer statistics, 2002 CA Cancer J Clin. 2005;55(2):74-108.

2. Brasil. Ministério da Saúde. Instituto Nacional de Câncer. Estimativa 2010: incidência de câncer no Brasil [online]. Rio de Janeiro: INCA, 2009. [citado 2011 Set 21] Disponível em: http:// www.inca.gov.br/estimativa/2010/estimativa20091201.pdf.

3. Elias D. Reflections and proposals for the standardization of lymphadenectomy for gastric carcinoma. Eur J Surg Oncol. 2000;26(1):6-10

4. Gama-Rodrigues JJ, Lopasso FP, Del Grande JC, Safatle NF, Bresciani C, Malheiros CA, et al., editores. Câncer do estômago. Aspectos atuais do diagnóstico e tratamento. São Paulo: Andrei; 2002.

5. Japanese Gastric Cancer Association. Japanese Classification of Gastric Carcinoma - 2nd English Edition -. Gastric Cancer. 1998; 1(1):10-24

6. Nakajima T. Gastric cancer treatment guidelines in Japan. Gastric Cancer. 2002;5(1):1-5.

7. Ichiyoshi Y, Tomoda M, Tomisaki S, Oda S, Ohno S, Maehara Y, et al. Macroscopic appearance and biological character of gastric cancer invading the muscularis propria. Hepatogastroenterology. 1996;43(9):553-9.

8. Nakamura K, Kamei T, Ohtomo N, Kinukawa N, Tanaka M. Gastric carcinoma confined to the muscularis propria: how can we detect, evaluate, and cure intermediate-stage carcinoma of the stomach? Am J Gastroenterol. 1999;94(8):2251-5.

9. Isozaki H, Fujii K, Nomura E, Mabuchi H, Nishiguchi K, Hara H, et al. Prognostic factors of advanced gastric carcinoma without serosal invasion ( $\mathrm{pT} 2$ gastric carcinoma). Hepatogastroenterology. 1999;46(28):2669-72.

10. Fotia G, Marrelli D, De Stefano A, Pinto E, Roviello F. Factors influencing outcome in gastric cancer involving muscularis and subserosal layer. Eur J Surg Oncol. 2004;30(9):930-4.

11. Komatsu S, Ichikawa D, Kurioka H, Kan K, Shioaki Y, Ueshima Y, et al. Prognostic and clinical evaluation of patients with $T 2$ gastric cancer. Hepatogastroenterology. 2005;52(63):965-8.

12. Otsuji E, Kuriu Y, Ichikawa D, Okamoto K, Hagiwara A, Yamagishi $\mathrm{H}$. Characteristics of gastric carcinoma invading the muscularis propria. J Surg Oncol. 2005;92(2):104-8.
13. Park SS, Kim CS, Mok YJ, Kim SJ, Kim HI. Gastric cancer confined to the muscularis propria: a possible candidate for laparoscopic surgery or adjuvant therapy. Scand J Gastroenterol. 2005:40(4):450-4.

14. Marchet A, Mocellin S, Ambrosi A, Morgagni P, Vittimberga G, Roviello $F$, et al. Validation of the new AJCC TNM staging system for gastric cancer in a large cohort of patients $(n=2,155)$ : focus on the T category. Eur J Surg Oncol. 2011;37(9):779-85.

15. Compton C, Sobin LH. Protocol for the examination of specimens removed from patients with gastric carcinoma: a basis for checklists. Members of the Cancer Committee, College of American Pathologists, and the Task Force for Protocols on the Examination of Specimens From Patients With Gastric Cancer. Arch Pathol Lab Med. 1998;122(1):9-14

16. Greene FL, Page DL, Fleming ID, Fritz A, Balch CM, Haller DG. American Joint Committee on Cancer. AJCC Cancer Staging Manual. 6th ed. New York: Springer-Verlag; 2002.

17. Washington K. 7th edition of the AJCC cancer staging manual: Stomach. Ann Surg Oncol. 2010;17(12):3077-9.

18. Ishigami S, Natsugoe S, Miyazono F, Hata Y, Uenosono Y, Sumikura $S$, et al. Clinical merit of subdividing gastric cancer according to invasion of the muscularis propria. Hepatogastroenterology. 2004;51(57):869-71.

19. Park do J, Kong SH, Lee HJ, Kim WH, Yang HK, Lee KU, et al. Subclassification of pT2 gastric adenocarcinoma according to depth of invasion (pT2a vs pT2b) and lymph node status (pN). Surgery 2007;141(6):757-63.

20. Lu Y, Liu C, Zhang R, Li H, Lu P, Jin F, et al. Prognostic significance of subclassification of pT2 gastric cancer: a retrospective study of 847 patients. Surg Oncol. 2008;17(4):317-22.

21. Tokunaga M, Hiki N, Fukunaga T, Ohyama S, Yamada K, Yamaguchi T. Better prognosis of T2 gastric cancer with preoperative diagnosis of early gastric cancer. Ann Surg Oncol. 2009;16(6):1514-9.

22. Sarela Al, Turnbull AD, Coit DG, Klimstra D, Brennan MF, Karpeh MS. Accurate lymph node staging is of greater prognostic importance than subclassification of the T2 category for gastric adenocarcinoma. Ann Surg Oncol. 2003;10(7):783-91.

23. Borrmann R. Geschwulste des margens. In: Henke F, Lubarsch O, editors. Handbuch spez pathol anat und histo. Berlim: SpringerVerlag; 1926. p. 864-71. 
24. Broders AC. The microscopic grading of cancer. Surg Clin North Am. 1941;21(4):947-62.

25. Kunisaki C, Shimada H, Nomura M, Matsuda G, Otsuka Y, Ono $H$, et al. Surgical outcome of serosa-negative advanced gastric carcinoma. Anticancer Res. 2004;24(5B):3169-75.

26. Nitti D, Marchet A, Mocellin S, Rossi GM, Ambrosi A, Mencarelli R. Prognostic value of subclassification of T2 tumours in patients with gastric cancer. Br J Surg. 2009;96(4):398-404.

27. Jiang CG, Wang ZN, Sun Z, Liu FN, Yu M, Xu HM. Clinicopathologic characteristics and prognosis of gastric cancer invading the subserosa. J Surg Oncol. 2010;102(7):737-41.

28. Ahn HS, Lee HJ, Hahn S, Kim WH, Lee KU, Sano T, et al. Evaluation of the seventh American Joint Committee on Cancer/International Union Against Cancer Classification of gastric adenocarcinoma in comparison with the sixth classification. Cancer. 2010;116(24):5592-8

29. Kim DH, Oh CA, Oh SJ, Choi MG, Noh JH, Sohn TS, et al. Validation of seventh edition AJCC gastric cancer staging modifications. J Surg Oncol. 2012;105(1):26-30.
30. Sasako M. What is reasonable treatment for gastric adenocarcinoma? J Gastroenterol. 2000;35 Suppl 12:116-20.

Recebido em 14/04/2012

Aceito para publicação em 10/06/2012

Conflito de interesse: nenhum

Fonte de financiamento: nenhum

Como citar este artigo:

Jucá PC, Lourenço L, Kesley R, Mello ELR, Oliveira IM, Corrêa JHS. Comparação da sobrevivência e dos fatores prognósticos em pacientes com adenocarcinoma gástrico T2 e T3. Rev Col Bras Cir. [periódico na Internet] 2012; 39(5). Disponível em URL: http://www.scielo.br/rcbc

Endereço para correspondência:

Patrícia Campos Jucá

E-mail: patriciajuca@ig.com.br 